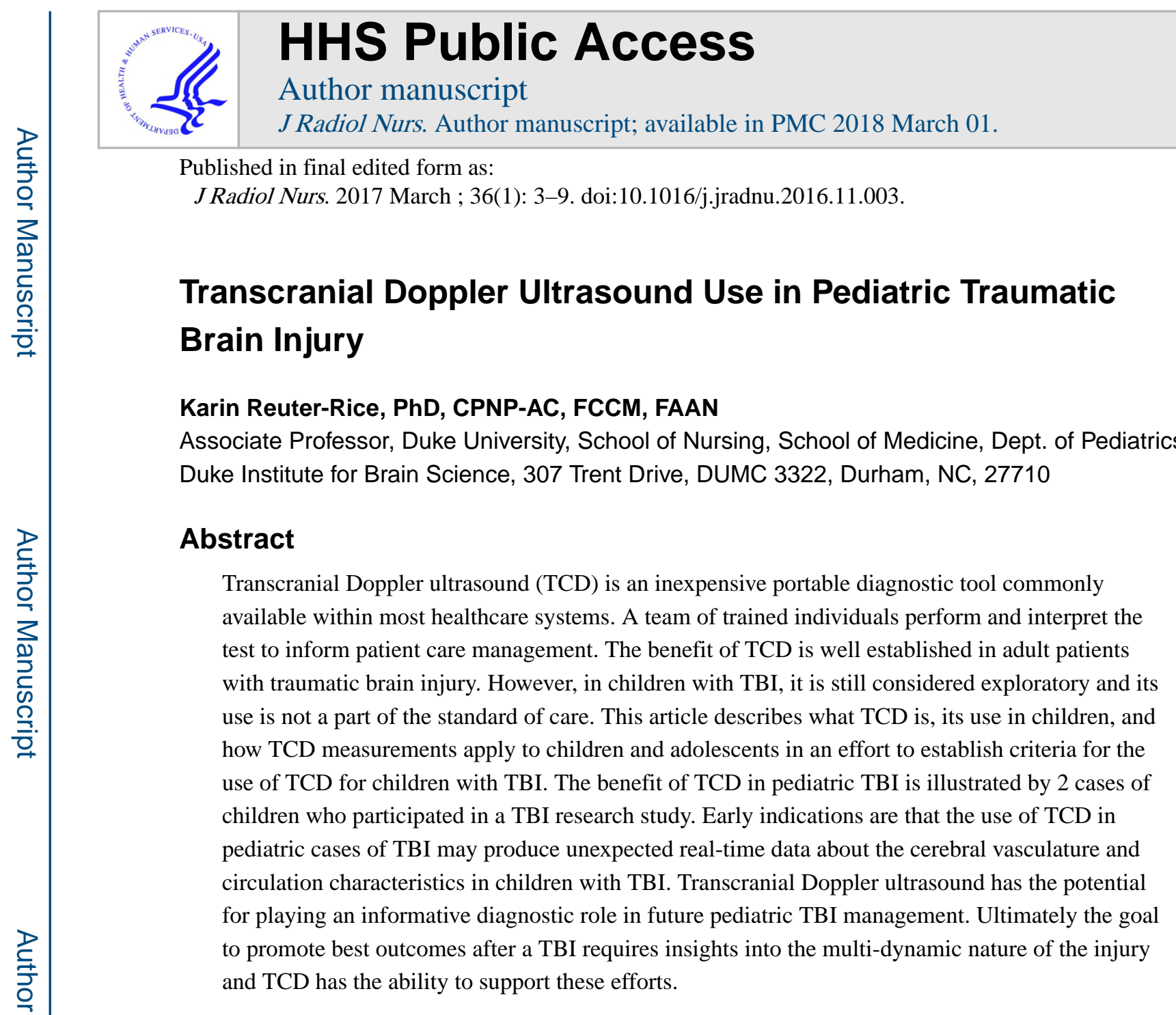

Keywords

cerebral flow velocity; children; head injury

\title{
Introduction
}

Transcranial Doppler ultrasound (TCD) is an inexpensive, non-invasive, portable, lowfrequency ultrasound that can measure cerebral blood flow velocities. The use of a Doppler ultrasound was first reported in the early 1960s, but it wasn't until 1982 when Rune Aaslid and his team described how to insonate the middle cerebral artery that TCD became a diagnostic measure for blood flow velocity (Aaslid, Markwalder \& Nornes, 1982; Coman, \& Popescu, 2015). Since that time, TCD has become standard practice in the evaluation of adult patients experiencing intracranial stenosis, such as acute stroke, sickle cell disease, and

Corresponding author/permanent address: 919-681-7647, karin.reuter-rice@ duke.edu.

This manuscript has not been previously presented.

Publisher's Disclaimer: This is a PDF file of an unedited manuscript that has been accepted for publication. As a service to our customers we are providing this early version of the manuscript. The manuscript will undergo copyediting, typesetting, and review of the resulting proof before it is published in its final citable form. Please note that during the production process errors may be discovered which could affect the content, and all legal disclaimers that apply to the journal pertain. 
subarachnoid hemorrhage. It has also been used to determine the presence or absence of collateral vascular flow and embolisms. It has the capability to assess cerebrovascular dynamics and function while also providing intraoperative monitoring in interventional neurodiagnostic procedures, cardiopulmonary bypass, and carotid endarterectomy (Markus, 2000; Newell, 1995). However, in children, TCD has been used most often in evaluations of sickle cell disease and cerebrovascular anomalies, and only recently in traumatic brain injury (TBI) (LaRovere \& O'Brien, 2015; Verlhac, 2011). As the indications for its clinical use expand, it behooves clinicians and researchers to familiarize themselves with TCD practice standards and guidelines, diagnostic interpretation, and common data reporting. Transcranial Doppler ultrasound plays a unique role in providing real-time information for patients at risk of cerebrovascular compromise.

\section{Transcranial Doppler Ultrasound}

Transcranial Doppler ultrasound has been recognized as a diagnostic device that allows the clinician to better understand the pathologic changes that affect cerebral circulation after injury. In adults with TBI, the majority of arteries within the Circle of Willis are measured (Table 1). However, in children the anterior (middle cerebral artery) and posterior (basilar artery) cerebral vessels are most commonly assessed and reported. Although the use of TCD in pediatric TBI is still considered experimental and it is not part of the standard of care, there is a growing body of literature. Most recent studies conducted using TCD in pediatric TBI have primarily focused on its use in the diagnosis of increased intracranial pressure, cerebral vasospasm, or hyperemia and trending of cerebral flow velocities (LaRovere \& O'Brien, 2015; LaRovere, O'Brien \& Tasker, 2015; O'Brien, Maa, Reuter-Rice, 2015; O’Brien, Maa, Yeates, 2015; O’Brien, Reuter-Rice, Khanna, Peterson \& Quinto, 2010).

The most common TCD devices are color Doppler ultrasounds, and they are located in radiology departments, neurodiagnostic labs, and some intensive care units. Doppler color flow systems assign a given color to the direction of flow; red is the flow toward the transducer, and blue is the flow away. The measurement of the cerebral arteries is determined by the systolic peak flow, the mean flow (mean flow velocity $=$ time - mean of the maximal velocity envelope curve) and the end diastolic peak flow. The derived calculated measures allow the determination of high-, normal-, and low-flow velocity states. Cerebral vasospasm is the state of higher-than-expected flow velocity and has been associated with poor outcomes after TBI (Lee, Martin, Alsina, McArthur, Zaucha, Hovda, \& Becker, 1997). In order to determine true cerebral vasospasm, a Lindegaard Ratio must be calculated. This ratio is based on the highest mean flow velocity in the middle cerebral artery divided by the extracranial internal carotid artery $(\mathrm{LR}=\mathrm{Vm} \mathrm{MCA} \div \mathrm{VmECICA})$ (Lindegaard, Nornes, Bakke, Sorteberg \& Nakstad (1989). This ratio must be three or greater to be considered true cerebral vasospasm; however, because normal cerebral flow velocities are different in adults and children, so are the definitions of cerebral vasospasm (Table 2) (O’Brien, 2015).

It is difficult to calculate blood flow volume based on mean flow velocities (FV) because the diameter of the artery is unknown. Therefore the Gosling pulsatility index (PI) is used to describe the TCD waveforms. The PI or Gosling equation is calculated by subtracting the 
end diastolic peak flow velocity from the systolic peak flow velocity and dividing the remainder by the mean flow velocity $\left(\mathrm{PI}=\left[\mathrm{FV}_{\text {sys }}-\mathrm{FV}_{\text {dia }}\right] \div \mathrm{FV}_{\text {mean }}\right.$. The calculated value is a ratio of the systolic peak flow velocity to end diastolic flow velocity. (De Riva, Budohoski, Smielewski, Kasprowwicz, Zweifel, Steiner, Reinhard, Fabregas, Pickard \& Czosnyka, 2012; Figaji, Zwane, Fieggen, Siesjo \& Peter, 2009).

The PI has been described as the distal cerebral vascular resistance and has been most associated with increased intracranial pressure (ICP) when PIs are recorded as higher than normal. However this has not been established with certainty in the pediatric TBI literature. In adults with TBI, a PI of one was associated with better functional outcomes after 6months that a PI of 1.56, which was associated with poorer outcomes such as death, vegetative state, and severe disability ((Moreno, Mesalles, Gener, Tomasa, Ley, Roca, \& Fernández-Llamazares, 2000). Yet when Figaji and his team examined the use of a PI of one to better predict increased ICP in children with severe TBI, they found PIs were unreliable non-invasive indicators. In a recent study PIs in children with severe TBI who had a PI threshold > 1.31 in the first 24 hours after injury had a higher association of increased ICP; thereafter, PI was not a trusted measure to solely manage ICP (O’Brien, Maa, \& ReuterRice, 2015).

Transcranial Doppler imaging refers to a generated pulse sound wave that moves from the transducer (probe) to the tissues. The sound waves bounce off of, in the case of TCD, moving red blood cells thereby creating echoes. The echoes are then sent back (reflected) to the ultrasound transducer to provide structural information and the speed and flow of blood (Figure 1). Location of cerebral arteries for TCD requires appropriate transducer placement (Table 3) over an "acoustic window." This is an area of the skull where the bones are thinner and allow for better penetration of the ultrasound beam. . The supine position is preferred for conducting a TCD (American College of Radiology, 2007), but in a child, a 30-degree head angle is allowed. In children, the depth for cerebral artery insonation is based on age and head circumference (Table 4), different from adults where these depths are stable regardless of age (Table 5). The direction of blood flow is the same for both. In children and adolescents, the mean flow velocity (Table 6) is commonly used for trending.

In 1988, Bode and Wais conducted a cross-sectional study to describe mean and standard deviation of flow velocities in children 0 days to 18 years of age (for a full description of these values please refer to Bode \& Wais, 1988). In the work, cerebral flow velocities are defined for systolic peak flow, mean flow, and end diastolic peak flow. Flow velocities have also been normed for sex and ages 4 to 16 (Table 7). Flow velocities are increasingly higher in the children from birth to 8 years of age, and to decline to adult values at age 18 (Bode \& Eden, 1989; Bode \& Wais, 1988; LaRovere \& O'Brien, 2015; O'Brien, 2015). Other studies have found that girls have higher MCA and BA flow velocities than boys (Tontisirin et al., 2006; Vavilala, Kincaid, Mauangman, Suz, Rozet \& Lam, 2005) and in children who are critically ill, ventilated and sedated have lower BA flow than healthy children (O'Brien, 2015). 


\section{Steps to Conducting a Transcranial Doppler Ultrasound for Pediatric Traumatic Brain Injury}

After a request for a TCD has been placed, the individual conducting the TCD enters all relevant patient data into the TCD software. Because the TCD is a portable ultrasound, the examination can be conducted at the bedside. Proper positioning of the patient's head in midline position will promote access of the bilateral anterior circulation. If medically safe, log rolling the patient on his or her side while maintaining the midline head position will allow access to the posterior circulation. In smaller children, the posterior circulation may often be accessed without turning them. If a cervical collar is in place it may be loosened, with the healthcare teams' permission, to promote access to the bilateral external carotids arteries and the basilar artery. While maintaining c-spine precautions, the transducer can be placed on the external carotid artery and foramen magnum. In school-age and adolescent patients, the opening in the cervical collar may allow access to the external carotid arteries without removing the collars.

Using a $2 \mathrm{MHz}$ Doppler transducer, the artery is insonated and interrogated (probe or walk the vessel) at $2 \mathrm{~mm}$ to $5 \mathrm{~mm}$ intervals along the entire length of the vessel. Transcranial Doppler settings can be adjusted to obtain the best quality flow images and capture. The gain setting should be maximized to easily define the flow velocity, and the power output should be as low as possible (ACR, 2007). Upon each interrogation or transducer move, a waveform analysis should be performed to ensure a good capture of the vessel is established (Figure 2). The procedure is then repeated for the opposite side of the head to capture the bilateral measurements.

The flow velocity and pulsatility index data is captured at each interval and recorded by the TCD machine (Figure 3). Interpretation of the TCD data is then performed, and all ultrasound images and interpretation should be included in the patient's healthcare record. The TCD findings in conjunction with other imaging and patient data are then discussed by the team to ascertain potential interventions. At this time, the TCD measurements have been used to manage pediatric post TBI stroke, but TCD use in the day-to-day management of cerebral blood flow velocities, such as in the case of cerebral vasospasm, is still experimental.

\section{The Use of Transcranial Doppler Ultrasound in Pediatric TBI Research}

The following cases are of 2 children who were enrolled as part of a prospective observational study $(\mathrm{N}=60)$ of children admitted to hospital for a mild, moderate or severe TBI. The purpose of the study was to examine the relationship of genomic and proteomic markers and their association with cerebral vasospasm and patient outcomes. The study was approved by the institutional IRB, and parental consent was obtained. The study protocol called for eight days of daily TCDs of the anterior (MCA) and posterior (BA) circulation. Additionally, blood, urine, and cerebrospinal fluid (if a ventricular drain was in place) were collected over a five-day period, and there was a one-time collection of buccal cells for Deoxyribonucleic acid (DNA). All subjects, ages 5 days to 15 years, were previously healthy and were from families that spoke either English or Spanish. All children received 
treated following standard TBI management guidelines (Kochanek, Carney, \& Adelson, 2012). Children remained on the study protocol for a maximum of eight days and were evaluated for functional status using the Glasgow Outcome Scale-Extended Pediatric (GOSE Peds) (Beers, Wisniewski, Garcia-Filion, Tian, Hahner, Berger, ... Adelson, 2012) at discharge and four to six weeks post-discharge. The healthcare team was blinded to the results, and the TCD was not part of the patient's record and did not inform patient management.

The following cases are of 2 children with isolated head injuries who experienced very similar hospitalizations. Both children did not require pre-hospital cardiopulmonary resuscitation; both were treated using the moderate to severe pediatric TBI guidelines in the emergency department and intensive care unit. Their hospital time course and their discharge and follow-up GOS-E Peds functional outcomes scores were also the same.

\section{Case 1}

A 4-year-old Caucasian female was admitted to the pediatric intensive care unit after an unwitnessed fall, later determined to be abusive head trauma. Her presenting Glasgow Coma Score (GCS) in the emergency room was 5 (severe TBI) and she was found to have a large right subdural hemorrhage. She was intubated and sedated and admitted to the pediatric intensive care unit. Her vital signs showed her to be afebrile with normal hemodynamics for age. Her right pupil was greater than her left, and she had an external ventricular drain placed to maintain an intracranial pressure of 14 and a cerebral perfusion pressure of 75 . She was placed on seizure prophylaxis and continuous electroencephalography, which did not indicate any seizure activity. She remained intubated on $30 \%$ oxygen with normal per-age ventilation settings. An arterial blood gas revealed a pH 7.39, paCO2 36, paO2 197, base excess -2 . Her hemoglobin was 7.6, hematocrit of 23, and platelet count of 181 . Her coagulation and electrolytes were all within normal limits for age.

Within 24 hours of admission to the intensive care unit consent was obtained and a TCD of the bilateral MCAs, extracranial internal carotids, and BA was conducted. Figure 4 demonstrates the mean flow velocities. Calculations of the TCD findings were based on flow velocities for age and gender and were interpreted by a physician blinded to the examination and the patient. The highest mean left MCA flow velocity was $75.1 \mathrm{~cm} / \mathrm{sec}$ at a depth of 40 $\mathrm{mm}$ and a PI of 1.01 with a ECICA 52.7. The highest mean right MCA flow velocity was $57.4 \mathrm{~cm} / \mathrm{sec}$ at a depth of $75 \mathrm{~mm}$ and a PI of 0.92 with a ECICA of 48.5 . The highest mean BA flow velocity was $62.4 \mathrm{~cm} / \mathrm{sec}$ at a depth of $85 \mathrm{~mm}$ and a PI of 0.78 . The right-side head injury led to transducing the right MCA beginning at a depth of $70 \mathrm{~mm}$ with termination depth of $90 \mathrm{~mm}$. When evaluating the child's PI values, the left MCA PI was highest at 1.09 at a depth of $50 \mathrm{~mm}$, the right MCA PI was 1.27 at $70 \mathrm{~mm}$ and the BA PI was 1.21 at 60 $\mathrm{mm}$.

Based on the Bode \& Wais (1988) expected cerebral blood flow velocities for this child's age, she should have a mean MCA flow velocity of $114 \mathrm{~cm} / \mathrm{sec}$ ( 2 standard deviations above norm) and a LR of $\geq 3$ to satisfy the criteria for anterior circulation cerebral vasospasm, but would need a mean BA flow velocity of $70 \mathrm{~cm} / \mathrm{sec}$ for a diagnosis of posterior circulation cerebral vasospasm. Yet, if we consider the adjusted values based on Tontisirin et al. (2007) 
study, she would need to satisfy a mean MCA flow velocity of $121 \mathrm{~cm} / \mathrm{sec}$ ( 2 standard deviations above norm) and a LR of $\geq 3$ to meet the criteria for anterior circulation cerebral vasospasm. She would also need a mean BA flow velocity of $90 \mathrm{~cm} / \mathrm{sec}$ for a diagnosis of posterior circulation cerebral vasospasm, which was not insonated. After calculating the left LR $(75.1 \div 52.7=1.43)$ and right $\operatorname{LR}(57.4 \div 48.5=1.18)$ both values are $\leq 3$ and would lead one to interpret that this child does not have cerebral vasospasm of the anterior circulation. All the PIs also fall within expected norms, although the right MCA PI of 1.27 is on the higher side. After hospitalization and treatment for her head injury this child was discharged with an assessment of a GOS-E Peds score of 4 indicating moderate functional outcome. Upon follow-up the patient had improved with a GOS-E Peds score of 2 or mild functional outcome.

\section{Case 2}

A 15-year-old Caucasian male was admitted to the pediatric intensive care unit after colliding with a parked car while riding a scooter. He was found without a helmet and transported to hospital by emergency services for a GCS of 10 (moderate TBI). In the emergency room he abruptly declined to a GCS of 3 (severe TBI). He was diagnosed with a large right subdural hemorrhage and intubated and sedated in the emergency department. His vital signs showed him to be afebrile with normal hemodynamics for age. His left pupil was greater than his right, and he had an external ventricular drain placed to maintain an intracranial pressure of 7 and a cerebral perfusion pressure of 74 . He was placed on seizure prophylaxis and continuous electroencephalography, which did not indicate any seizure activity. He remained intubated on $40 \%$ oxygen with normal per-age ventilation settings. An arterial blood gas revealed a pH 7.45, paCO2 34, paO2 165, base excess 0 . His hemoglobin was 8.8 , hematocrit of 29 with a platelet count of 184 . His coagulation and electrolytes were all within normal limits for age.

Within 24 hours of admission to the intensive care unit consent was obtained and a TCD of the bilateral MCAs, extracranial internal carotids, and BA was conducted. Figure 5 demonstrates the mean flow velocities. Calculations of the TCD findings were based on flow velocities for age and gender and were interpreted by a physician blinded to the examination and the patient. The highest mean left MCA flow velocity was $159 \mathrm{~cm} / \mathrm{sec}$ at a depth of 60 $\mathrm{mm}$ and a PI of 0.78 with an ECICA of 46.6. The highest mean right MCA flow velocity was $162 \mathrm{~cm} / \mathrm{sec}$ at a depth of $60 \mathrm{~mm}$ and a PI of 0.64 with an ECICA of 51.2. The highest mean BA flow velocity was $86.2 \mathrm{~cm} / \mathrm{sec}$ at a depth of $100 \mathrm{~mm}$ and a PI of 0.72 . When evaluating his PI values, the left MCA PI was highest at 0.92 at a depth of $55 \mathrm{~mm}$, the right MCA PI was 0.82 at $64 \mathrm{~mm}$ and the BA PI was 0.88 at $95 \mathrm{~mm}$.

Based on the Bode \& Wais (1988) expected cerebral blood flow velocities for this child's age, he should have a mean MCA flow velocity of $103 \mathrm{~cm} / \mathrm{sec}$ (2 standard deviations above norm) and a LR of > 3 to satisfy the criteria for anterior circulation cerebral vasospasm and he would need a mean BA flow velocity of $62 \mathrm{~cm} / \mathrm{sec}$ for a diagnosis of posterior circulation cerebral vasospasm. Yet, if we consider the adjusted values based on gender and age Vavilala et al. (2005) study, he would need to satisfy a mean MCA flow velocity of $104 \mathrm{~cm} / \mathrm{sec}$ ( 2 standard deviations above norm) and a LR of $\geq 3$ to meet the criteria for 
anterior circulation cerebral vasospasm. He would also need a mean BA flow velocity of $75 \mathrm{~cm} / \mathrm{sec}$ for a diagnosis of posterior circulation cerebral vasospasm. Although the PIs fall within expected norms, the bilateral MCAs and BA mean flow velocities are well over the age/gender norms. The higher than expected BA mean flow velocity indicated cerebral vasospasm of the posterior circulation. When the left sided LR $(159 \div 46.6=3.41)$ and right LR $(162 \div 51.2=3.16)$ were calculated both values demonstrated a LR of $>3$ and would lead one to interpret that this child also had cerebral vasospasm of the anterior circulation. After hospitalization and treatment for his head injury he was discharged with an assessment of a GOS-E Peds score of 4 indicating moderate functional outcome and upon follow-up he showed improvement with a GOS-E Peds score of 2 or mild functional outcome.

\title{
Case Impressions
}

The cases illustrate the use of TCD in 2 severely brain injured children admitted to hospital for management. The TCD assessed real-time cerebral flow velocities, which at the time of study reveal that one patient was experiencing cerebral vasospasm whereas the other was not. Regardless of their age, sex, stable intracranial and a cerebral perfusion pressures, afebrile state and age appropriate hemodynamics these patients cerebral flow velocities could not have been foreseen as different. The TCD, although used in these cases for research, provided insights into these patient's anterior and posterior circulation; insights that were unappreciated without the use of the TCD. Although limited treatment options exist in adult TBI for cerebral vasospasm, they do not exist in children with TBI because we lack the information needed to better understand the cerebral circulation after injury and what triggers cerebral vasospasm.

\section{Conclusion}

Transcranial Doppler ultrasound is an inexpensive portable diagnostic tool commonly available in most healthcare facilities with trained individuals who can perform and interpret the test. The use of TCD has been well established in adult patients with TBI, however in children, it is still considered exploratory. The inclusion of TCD in pediatric TBI research would define the timing of, potential properties of, flow characteristics, and common data elements of cerebral vasospasm could be achieved by. It would also establish TCD as a diagnostic tool that has the capability to contribute real-time data about the cerebral vasculature. This real-time data has the potential to shape future management of children with TBI and make an impact on their outcomes.

\section{Acknowledgments}

\author{
Funding/Grant acknowledgement:D \\ This work was supported by the National Institutes of Health [NINR 1P30 NR014139-01 Adaptive Leadership for \\ Cognitive \\ Affective Symptom Science ADAPT Institute of Nursing \\ Research Center of Excellence; 2012-2015]; and by the Robert Wood Johnson Foundation as a Nurse Faculty \\ scholar project [71244; 2013-2016].
}


The author would like to acknowledge Ms. Jane Shealy, Editor, Duke University School of Nursing for her editorial assistance.

\section{References}

Aaslid R, Markwalder T, Nornes H. Noninvasive transcranial Doppler ultrasound recording of flow velocity in basal cerebral arteries. Journal of Neurosurgery. 1982; 57(6):769-74. DOI: 10.3171/jns. 1982.57.6.0769 [PubMed: 7143059]

Alexandrov A, Sloan M, Wong L, Douville C, Razumovsky A, Koroshetz W, Kaps M, Tegeler C. for the American Society of Neuroimaging Practice Guidelines Committee. Practice standards for transcranial Doppler ultrasound: Part 1 - test performance. J Neuroimaging. 2006; 17(1):11-18. DOI: $10.1111 / \mathrm{j} .1552-6569.2006 .00088 . x$

American College of Radiology. ACR-AIUM Practice Guideline for the Performance of Transcranial Doppler Ultrasound for Adults and Children. 2007. p. 1-5.(Resolution 33)http://www.acr.org/ guidelines

Bathala L, Mehndiratta M, Sharma V. Transcranial Doppler: Technique and common findings (part 1). Ann Indian Acad Neurol. 2013; 16(2):174-179. DOI: 10.4103/0972-2327.112460 [PubMed: 23956559]

Beers SR, Wisniewski SR, Garcia-Filion P, Tian Y, Hahner T, Berger RP, ... Adelson PD. Validity of a Pediatric Version of the Glasgow Outcome Scale-Extended. Journal of Neurotrauma. 2012; 29(6): 1126-1139. DOI: 10.1089/neu.2011.2272 [PubMed: 22220819]

Bode H, Eden A. Transcranial Doppler sonography in children. J Child Neurology. 1989; 4(suppl 1):s68-s76. DOI: $10.1177 / 0883073889004001111$

Bode H, Wais U. Age dependence of flow velocities in basal cerebral arteries. Archives of Disease in Childhood. 1988; 63:606-611. [PubMed: 3389890]

Coman IM, Popescu BA. Shigeo Satomura: 60 years of Doppler ultrasound in medicine. Cardiovascular Ultrasound. 2015; 13:48.doi: 10.1186/s12947-015-0042-3 [PubMed: 26699126]

De Riva N, Budohoski K, Smielewski P, Kasprowwicz M, Zweifel C, Steiner L, Reinhard M, Fabregas N, Pickard J, Czosnyka M. Transcranial Doppler pulsatility index: What it is and what it isn't. Neurocrit Care. 2012; 17:58-66. DOI: 10.1007/s12028-012-9672-6 [PubMed: 22311229]

Edelman, S. Doppler in Understanding Ultrasound Physics. 3. Woodlands, TX: ESP; 2007. p. 293-328.

Figai A, Zwane E, Fieggen G, Siesjo P, Peter J. Transcranial Doppler pulsatility index is not reliable indicator or intracranial pressure in children with severe traumatic brain injury. Surgical Neurology. 2009; 72:389-394. DOI: 10.1016/j.surneu.2009.02.012 [PubMed: 19608224]

Kochanek PM, Carney N, Adelson PD. Guidelines for the acute medical management of severe traumatic brain injury in infants, children, and adolescents-second edition (Pediatric Critical Care Medicine (2012). Pediatric Critical Care Medicine. 2012; 13(2):252.doi: 10.1097/PCC. $0 \mathrm{~b} 013 \mathrm{e} 31823 \mathrm{f} 435 \mathrm{c}$

LaRovere K, O’Brien N. Transcranial Doppler sonography in pediatric neurocritical care: A review of clinical applications and case illustrations in the pediatric intensive care unit. J Ultrasound Med. 2015; 34:2121-2132. DOI: 10.7863/ultra.15.02016 [PubMed: 26573100]

LaRovere K, O'Brien N, Tasker R. Current opinion and use of Transcranial Doppler ultrsonography in traumatic brain injury in pediatric intensive care unit. J Neurotrauma. 2016; 33:1-10. DOI: 10.1089/neu.2015.4344 [PubMed: 25962860]

Lee J, Martin N, Alsina G, McArthur D, Zaucha K, Hovda D, Becker D. Hemodynamically significant cerebral vasospasm and outcome after head injury: A prospective study. Journal of Neurosurgery. 1997; 87(2):221-233. [PubMed: 9254085]

Lindegaard K, Nornes H, Bakke SJ, Sorteberg W, Nakstad P. Cerebral vasospasm diagnosis by means of angiography and blood velocity measurements. Acta Neurochir. 1989; 100:12-24. [PubMed: 2683600]

Markus H. Transcranial Doppler ultrasound. British Medical Bulletin. 2000; 56(2):378-388. [PubMed: 11092087]

Newell DW. Transcranial Doppler measurements. New Horizons (Baltimore, Md). 1995; 3(3):423430. 
Moreno J, Mesalles E, Gener J, Tomasa A, Ley A, Roca J, Fernández-Llamazares J. Evaluating the outcome of severe head injury with transcranial Doppler ultrasonography. Neurosurg Focus. 2000; 8(1):1-7.

O'Brien N. Reference values for cerebral flow velocities in critically ill, sedated children. Childs Nerv Syst. 2015; 31:2269-2276. DOI: 10.1007/s00381-015-2873-5 [PubMed: 26285761]

O'Brien N, Maa T, Reuter-Rice K. Noninvasive screening for intracranial hypertension in children with acute, severe traumatic brain injury. J Neurosurgery: Pediatrics. 2015; 16(4):420-425. DOI: 10.3171/2015.3.PEDS14521 [PubMed: 26140576]

O'Brien N, Maa T, Yeates K. The epidemiology of vasospasm in children with moderate-to-severe traumatic brain injury. Critical Care Medicine. 2015; 43(3):674-685. DOI: 10.1097/CCM. 0000000000000745 [PubMed: 25479116]

O’Brien N, Reuter-Rice K, Khanna S, Peterson B, Quinto K. Vasospasm in children with traumatic brain injury. Intensive Care Med. 2010; 36(4):680-7. DOI: 10.1007/s00134-009-1747-2 [PubMed: 20091024]

Tontisirin N, Muangman S, Suz P, Pihoker C, Fisk D, Moore A, Lam A, Vavilala M. Early childhood gender differences in anterior and posterior cerebral blood flow velocity and autoregulation. Pediatrics. 2006; 119(3):e610-615. DOI: 10.1542/peds.2006-2110

Vavilala M, Kincaid S, Muangman S, Suz P, Rozet I, Lam A. Gender differences in cerebral flow velocity and autoregulation between anterior and posterior circulations in health children. Pediatric Research. 2005; 58(3):574-578. DOI: 10.1203/01.PDR.0000179405.30737.0F [PubMed: 16148076]

Verlhac S. Transcranial Doppler in children. Pediatr Radiol. 2011; 41(Suppl 1):S153-S165. DOI: 10.1007/s00247-011-2038-y [PubMed: 21523592] 


\section{Highlights}

- Transcranial Doppler ultrasound is a non-invasive, portable, economic diagnostic test that can provide real-time information.

- Transcranial Doppler ultrasound is useful for screening and diagnostic trending of cerebral circulation.

- In children with traumatic brain injury, insonation of the middle and basilar arteries provides information about flow velocities in the anterior and posterior cerebral circulation. 


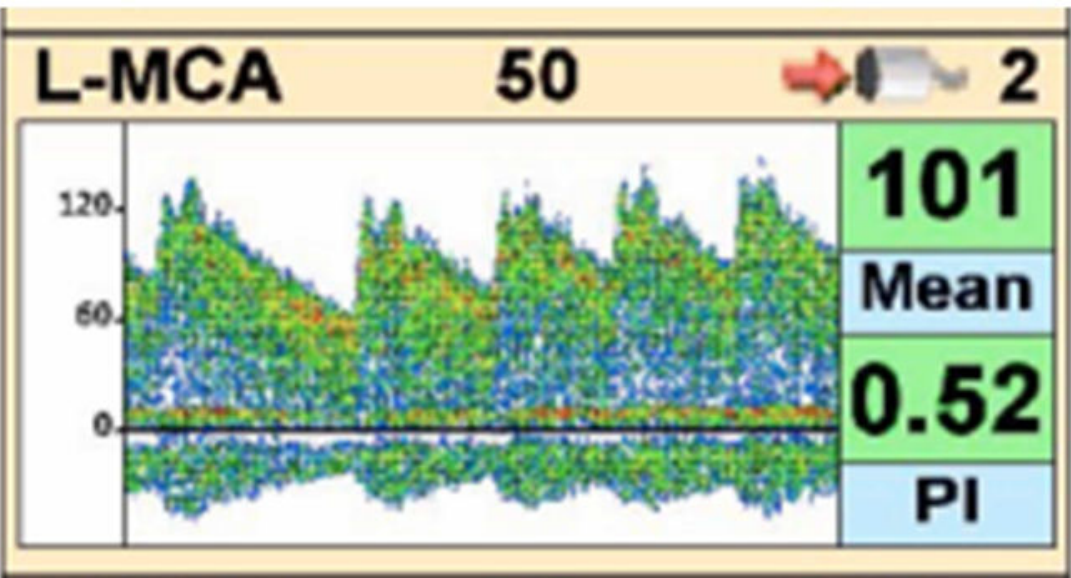

Figure 1.

Example of a mean flow velocity of the left middle cerebral artery (L-MCA) at a depth of $50 \mathrm{~mm}$ with a flow of $101 \mathrm{~cm} / \mathrm{sec}$ and a pulsitility index (PI) of 0.52 . 

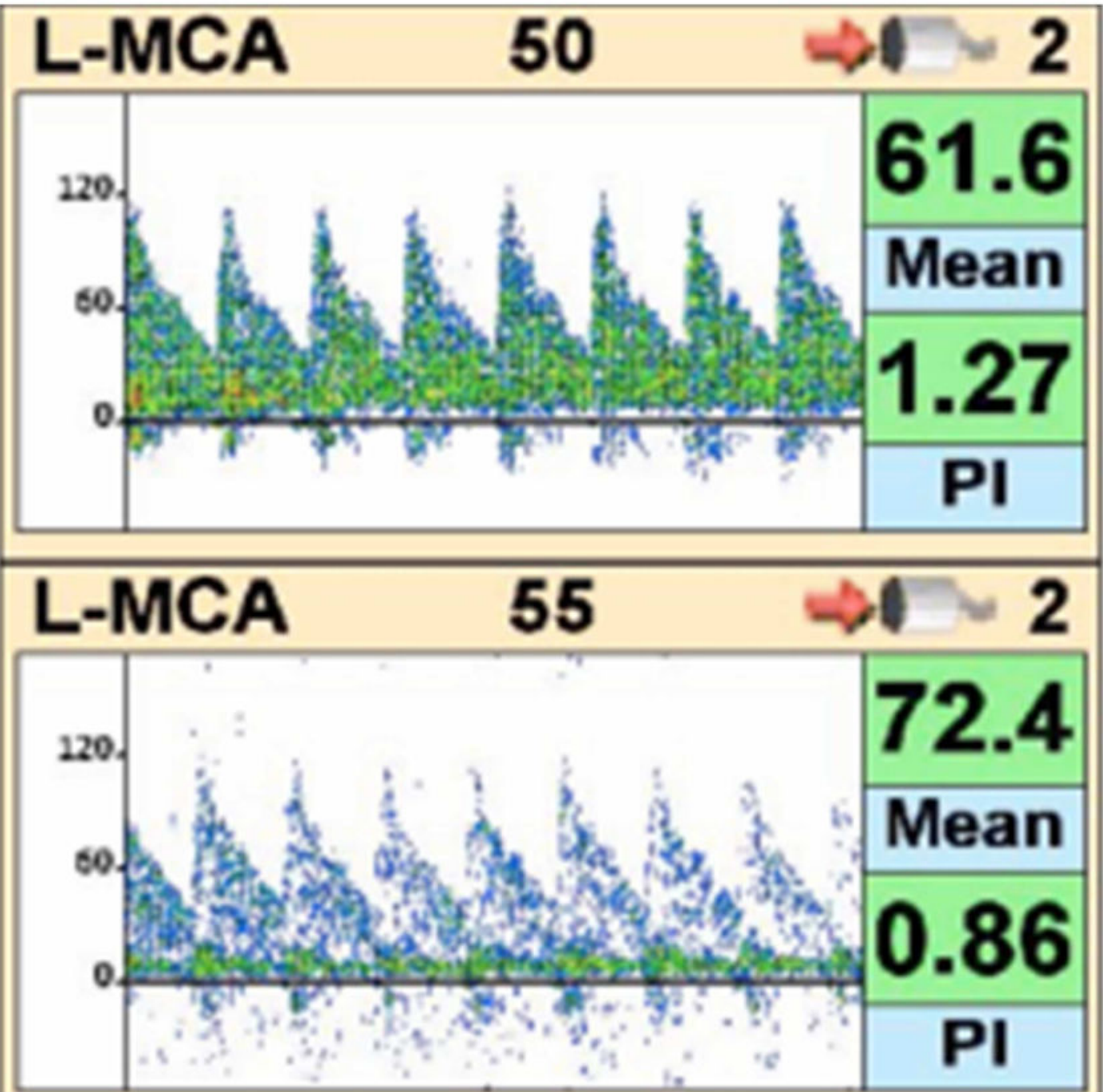

Figure 2.

A change in the quality of the waveform when the left middle cerebral artery (L-MCA) is interrogated from a depth of $50 \mathrm{~mm}$ to $55 \mathrm{~mm}$. 


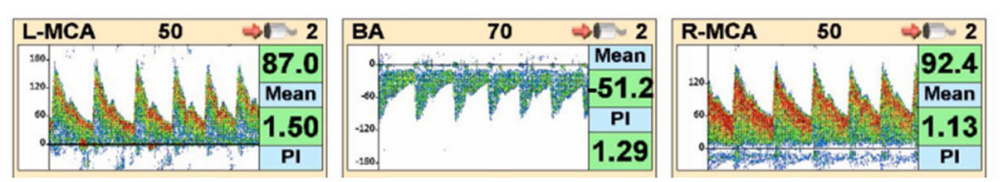

Figure 3.

Example of mean flow velocities of the left middle cerebral artery (L-MCA), the basilar artery (BA) and the right middle cerebral artery (R-MCA). The color Doppler flow tracings demonstrate a L-MCA at a velocity of $87.0 \mathrm{~cm} / \mathrm{sec}$ at a depth of $50 \mathrm{~mm}$ and a pulsatility index (PI) 1.50 , BA velocity of $51.2 \mathrm{~cm} / \mathrm{sec}$ at a depth of $70 \mathrm{~mm}$ with a PI of 1.29 and a R-MCA velocity of $92.4 \mathrm{~cm} / \mathrm{sec}$ at a depth of $50 \mathrm{~mm}$ with a PI 1.13 . 

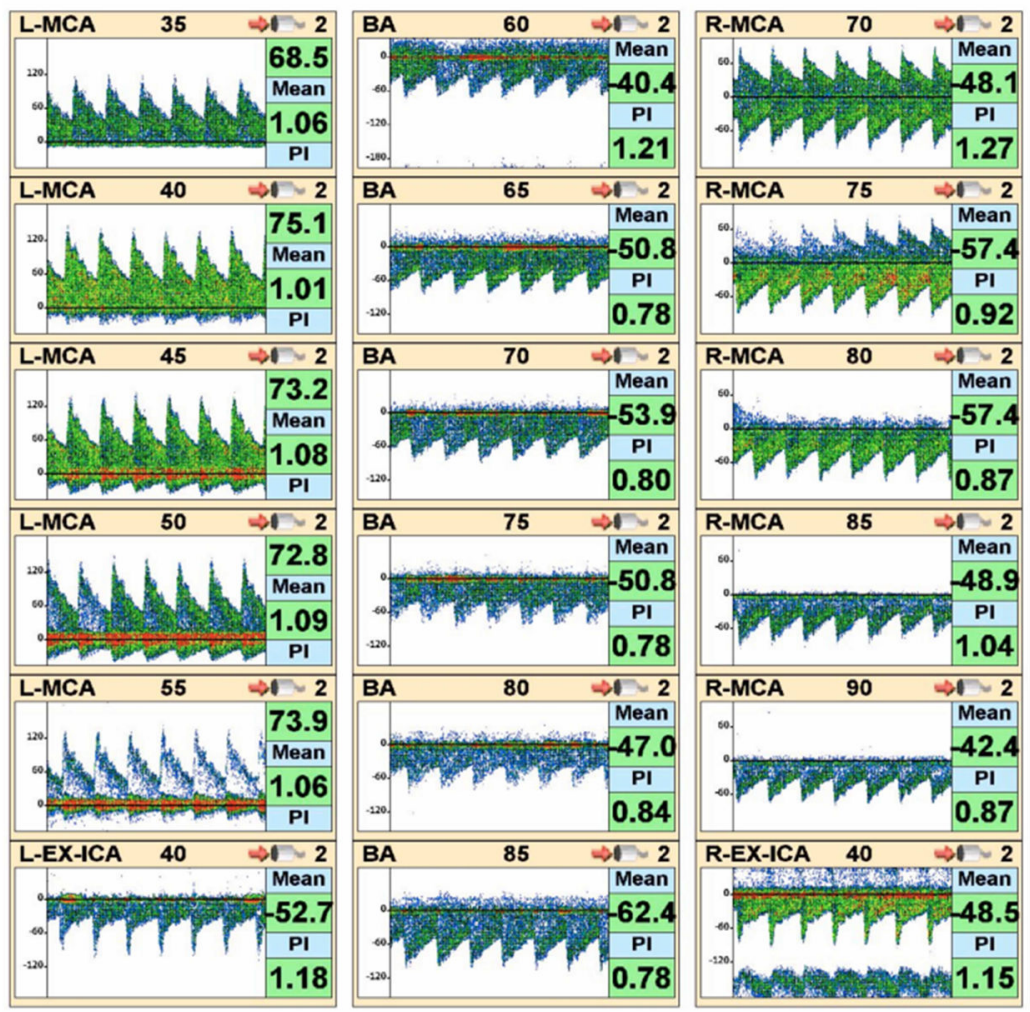

Figure 4.

First 24 hour TCD results in a 4 year old girl admitted to hospital for a severe TBI. 

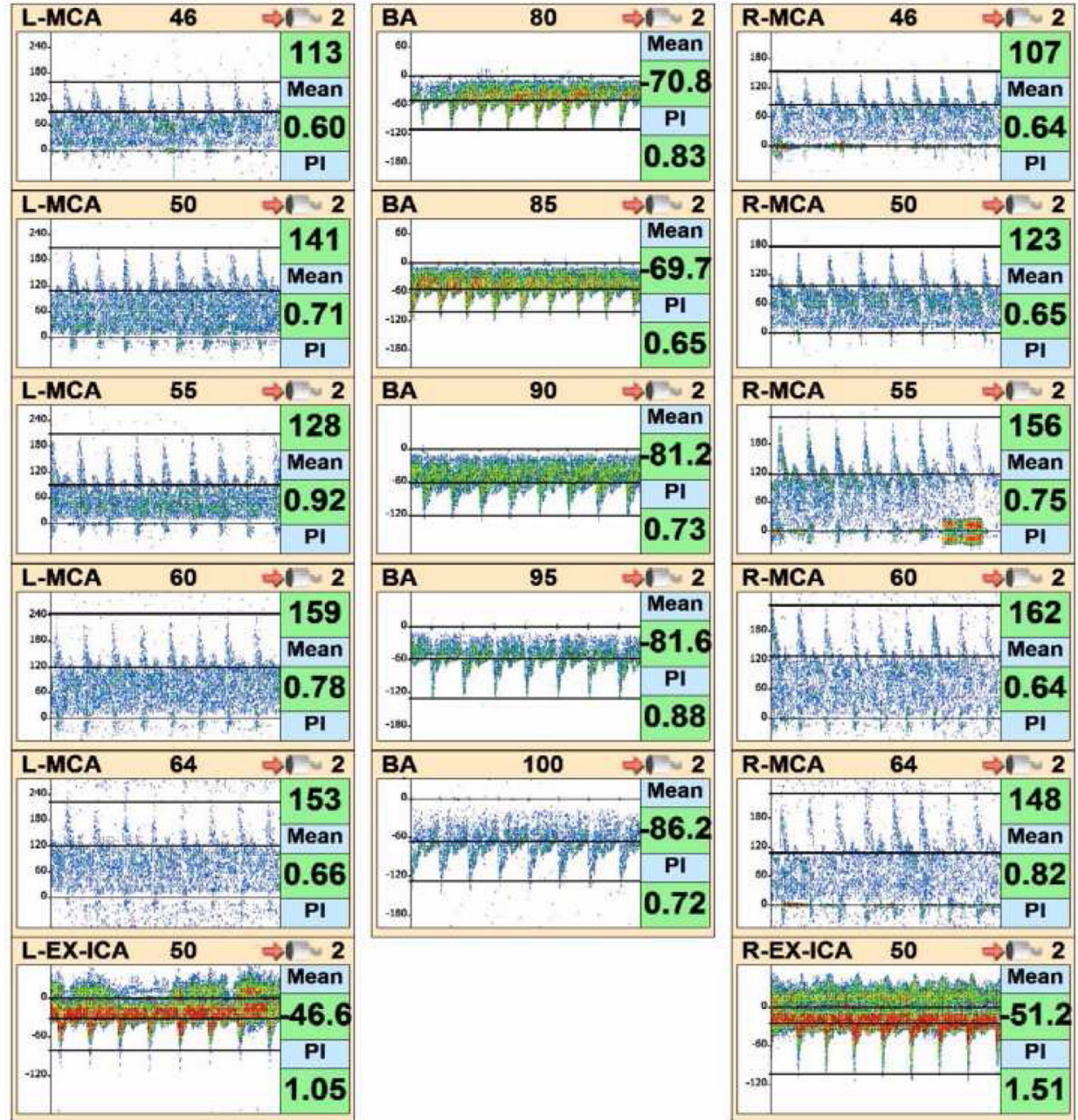

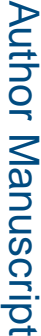

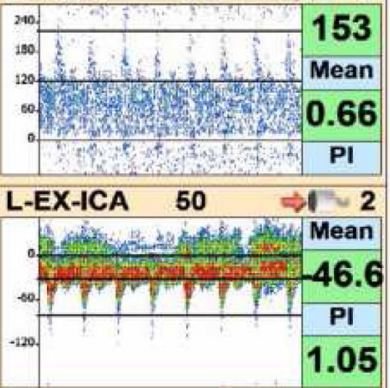

Figure 5.

First 24 hour TCD results in a 15-year-old boy admitted to hospital for a severe TBI. 


\section{Table 1}

Cerebral Arteries Measured by TCD

\begin{tabular}{|l|l|}
\hline Vessel & Abbreviation \\
\hline Middle Cerebral Artery & MCA \\
\hline Anterior Cerebral Artery & ACA \\
\hline Posterior Cerebral Artery & PCA \\
\hline Terminal Internal Carotid Artery & TICA \\
\hline Internal Carotid Artery (Siphon) & ICA \\
\hline Ophthalmic Artery & OA \\
\hline Vertebral Artery & VA \\
\hline Basilar Artery & BA \\
\hline
\end{tabular}


Table 2

Definitions of Cerebral Vasospasm in Adults and Children

\begin{tabular}{|l|l|l|}
\hline Individual & Anterior Cerebral Vasospasm & Posterior Cerebral Vasospasm \\
\hline Adult & MCA $>120 \mathrm{~cm} / \mathrm{sec} A N D \mathrm{LR} \geq 3$ & $\mathrm{BA}>90 \mathrm{~cm} / \mathrm{sec}$ \\
\hline Pediatric & MCA $\geq 2 \mathrm{SD}$ above normal for age AND LR $\geq 3$ & $\geq 2 \mathrm{SD}$ above normal for age \\
\hline
\end{tabular}

BA: Basilar artery; LR: Lindegarrd Ratio; MCA: Middle cerebral artery 
Table 3

\begin{tabular}{|c|c|}
\hline Acoustic Window & Artery Insonation \\
\hline \multirow[t]{3}{*}{ Trans-temporal } & Middle cerebral \\
\hline & Anterior cerebral \\
\hline & Posterior cerebral \\
\hline \multirow[t]{2}{*}{ Trans-orbital } & Ophthalmic \\
\hline & Internal carotid \\
\hline \multirow[t]{2}{*}{ Sub-occipital } & Vertebral \\
\hline & Basilar \\
\hline External carotid & Extracranial carotid \\
\hline
\end{tabular}




\section{Table 4}

Pediatric TCD Insonation Depth (mm)

\begin{tabular}{|l|l|l|l|l|}
\hline Head Diameter $(\mathbf{c m})$ & Proximal MCA & ICA & ACA & PCA \\
\hline 12 & $30-54$ & $50-54$ & $50-58$ & $40-60$ \\
\hline 13 & $30-58$ & $52-58$ & $52-62$ & $42-66$ \\
\hline 14 & $34-62$ & $56-64$ & $56-58$ & $46-70$ \\
\hline
\end{tabular}

Adapted from Alexandrov et al., 2006

MCA: middle cerebral artery; ICA: internal carotid artery; ACA: anterior cerebral artery; PCA: posterior cerebral artery 
Table 5

Expected TCD Insonation Depth for Adults and Flow Direction

\begin{tabular}{|l|l|l|}
\hline Artery & Depth $(\mathbf{m m})$ & Direction of Flow \\
\hline MCA & $40-65$ & Towards \\
\hline ACA & $60-75$ & Away \\
\hline PCA & $55-75$ & Bidirectional \\
\hline BA & $80-105$ & Away \\
\hline
\end{tabular}

MCA: middle cerebral artery; ACA: anterior cerebral artery; PCA: posterior cerebral artery; BA: basilar artery 


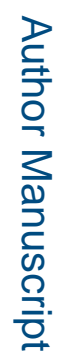
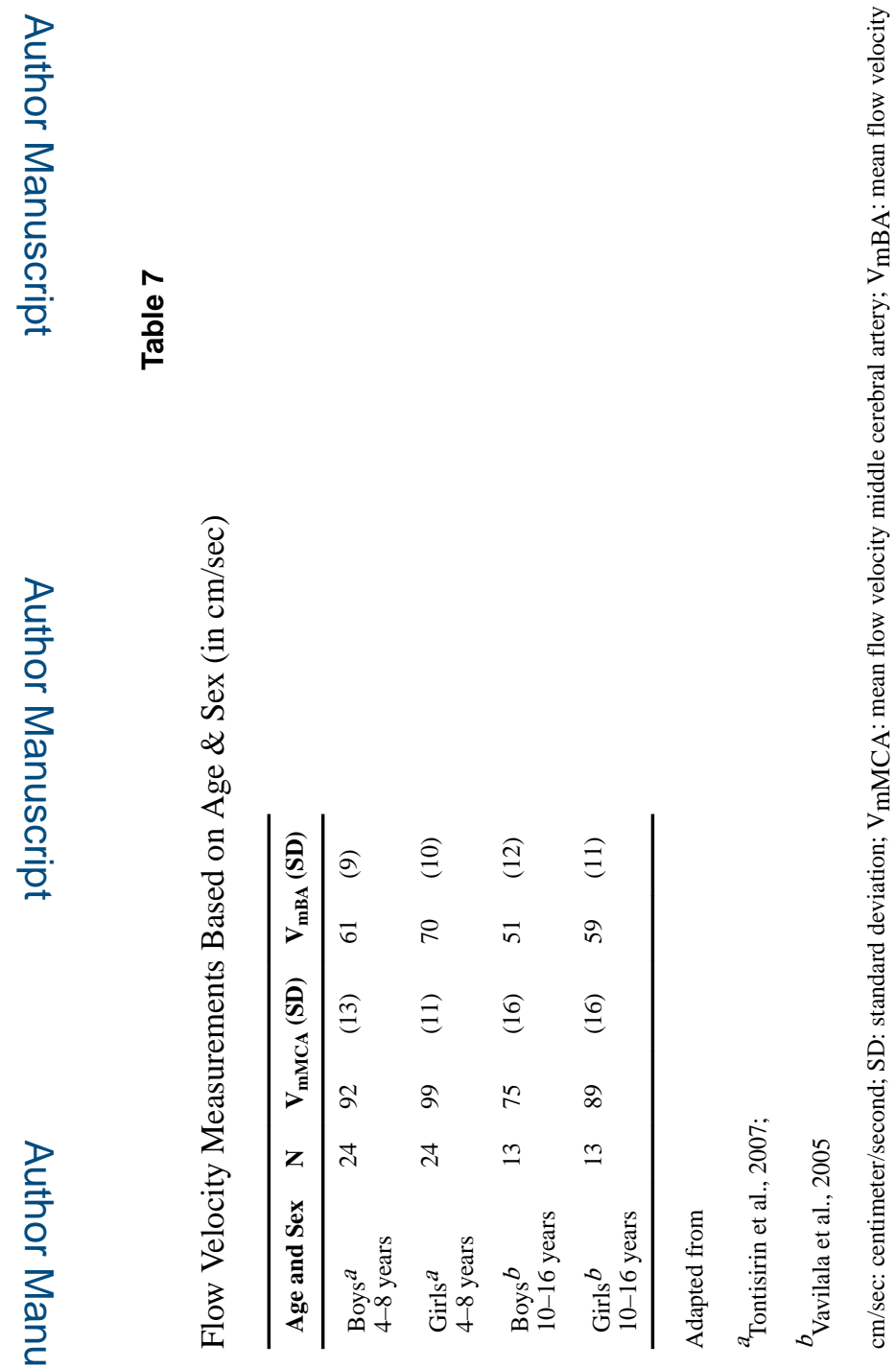